\title{
THE DEVELOPMENT OF THE FLAT-KNITTED SHAPED UPPERS BASED ON ERGONOMICS
}

\author{
Zhiwen Lu, Gaoming Jiang, Honglian Cong, Xi Yang \\ Engineering Research Center for Knitting Technology, Jiangnan University, Wuxi 214122, China \\ E-mail: Jiang G. jiang@526.cn
}

\begin{abstract}
:
To achieve the efficiency and specification of the flat-knitted uppers design, the basic patterns of uppers are made from shoe lasts based on the research on the characteristics of human's feet and wearability requirements on uppers. The knitting technology for half-shaped and fully shaped uppers was formed after the shear deformation of basic pattern and combination with flat knitting technology. As regards to the functional requirements on key parts of uppers, the structures of flat-knitted shaped uppers were intensively analysed and studied, dividing them into two categories (functional structure and decorative structure), discussing the knitting methods and advantages of different structure, and finally experimentally proving that the planar pattern of flat knitted uppers can apply to the design of flat-knitted uppers and achieve the combination of functionality and artistry of sneakers after combining with structural changes, with a great significance on the achievement of the efficient production of uppers and the enhancement of its commercial value.
\end{abstract}

\section{Keywords:}

flat-knitted upper, sports shoes, shaping technique,

\section{Introduction}

Flat-knitted shaped uppers refers to the upper which were produced by the flat knitting machine. With the improvement of living standards, people have a higher demand for the comfort of sportswear, such as lightweight, functional and other decorative effects [1]. Flat-knitted shaped uppers has these excellent properties and also with a high degree of forming, knitted with dyed yarn and having no dying process, present plentiful colours and structure pattern. An effective means to reduce processing and pollution and improve the product integrity have become hot spots for research.

Flat knitting technology has a great advantage in forming the seamless garment. The knitting techniques have been considered as an innovative process. Flat knitting technology provides a variety of advantages in knitting production such as savings in cost and time, higher productivity, quick response production and other advantages [2]. Flat knitted fabric have been playing a predominant role in the area of technical textiles [3]. They have been knitted into net shapes and have advantages such as high conformability/drape ability to accommodate complex shape moulds [4]. Flat-knitted spacer fabrics offer a strong potential for complex shape preforms which could be used to manufacture composites with reduced waste and shorter production times [5]. There are other scholars who are studying computer-aided design system and the basic knitting technology of three-dimensional knitwear in the flat knitting machine [6]. Now flat knitted uppers products are also developing rapidly, has been pushed to the market successfully, but the professional theoretical research is lacking.
This research presents the basic patterns of uppers based on the research in characteristics of human's feet, and then the uppers knitting technology is formed through a combination with flat knitting technology. As regards to different requirements on uppers, the functional structure and decorative structure were developed, the knitting methods and advantages of different structure were discussed, which filled a gap in flat knitting uppers forming theory and finally experimentally proved that, with a great significance on the achievement of efficient production of uppers.

\section{Human foot anatomy and upper's properties}

The foot is an important organ in the body, has a certain morphological structure, physiological function and characteristics of motion. Before the design of upper pattern, it is essential to study the foot basic structure and functions. Many experts and scholars were of the opinion that a pair of qualified sport shoes should meet a number of requirements, such as lightweight, convenience, more flexible; antiskid, shock absorption, reducing energy consumption, etc.[7]. Upper material needs to have a good moisture absorption to keep shoes dry the cavity. Also can provide the footwear with good covering and supporting capacity, and give the wearer a comfortable dress environment [8].

\subsection{Human foot anatomy and function}

Human foot has 26 pieces of bones, dozens of joints. According to the different functions and locations, human bones in the feet can be divided into three categories: phalanges, metatarsus 
and tarsus bones [9]. Metatarsals and phalanges of each section constitute a toe metatarsal joint, it is the part of the foot that is used for frequent activity. It is affected by high pressure in the process of running and jumping, so this place is best to seamless during upper pattern design.

Ankle is composed of tibia, fibula and talus, it can move in multiple directions, but excessive activity may cause damage [10], so good protection and support are very important for the ankle in the design of sports shoes. As shown in Figure 1, arch is an important component of the foot, whose main function is to guarantee the stability when standing and a cushioning effect, while jumping or falling.

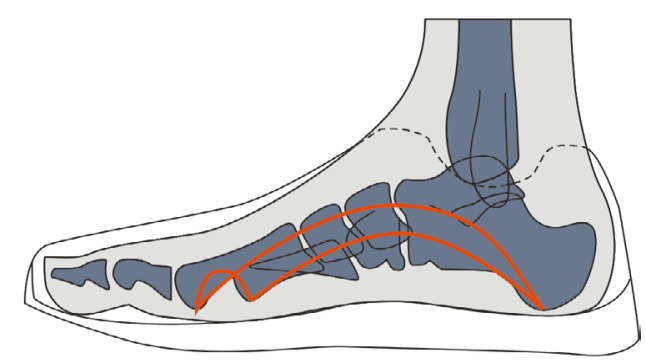

Figure 1. Diagram of human foot

Physiology refers to the organ's ability to work in a normal state. Physiology of the foot includes regulating temperature, secreting sweat, evaporation and other. Because of the different states, season and human perspiration, foot perspiration status changes, mainly reflecting the forefoot and sole sweating, which has more glands. So the shoe's warmth retention property should be given attention during the design process, while also adding ventilation holes in the upper to fulfil the shoes chamber atmospheric requirements.

\subsection{Upper wearability}

Comfort and breath ability have close relationship with the material and the weight of the shoe, the lighter the weight, the higher the comfort. Studies showed that a $1 \%$ increase in the weight of the shoe and a $3 \%$ to $10 \%$ increase in human energy consumption [11]. Shoe upper plays a wrapping and supporting role for foot in the process of walking and sports. It not only helps to keep warm while also providing a guarantee of safety and stability [12]. With the development of fashion industry, the combination of sports and fashion became inevitable, personalised, fashion orientated, customised to become the direction for future sports products, sport shoes as mouth pieces of healthy and vigorous, in addition to meeting the wearability, but also to have some decorative function.

\section{Design and implementation of flat-knitted shaped uppers}

\subsection{Shoe last and basic pattern of flat-knitted shaped uppers}

The shoe last, depending on foot type, can be deemed as the foot model but not just a replica of the foot [13]. It determines the shoe shape and style as well as the fitness and wearer's comfort. Therefore, in terms of shoe last design, it is crucial to collect the precise data of a human's foot. Based on the collected foot size data, people organize and correct the shoe last parameters. Since the shoe's last surface is curved in a three-dimensional space, freely extending in multiple directions, copy down the area and the information of ups and downs for the shoe last surface as far as possible. The flattening process is shown in Figure 2. First, notches were cut in the toe cap, then uniformly dispersed in the folded part in the direction of arrow, and finally get the planar shoe last shape.

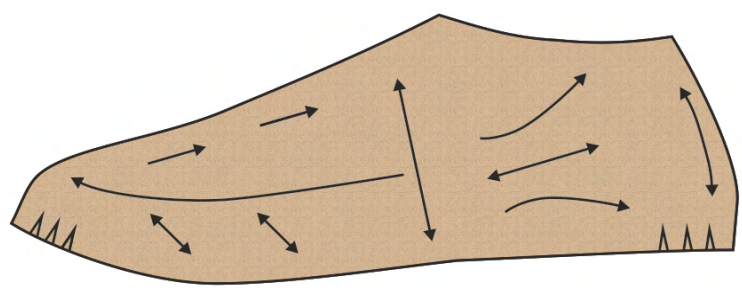

Figure 2. Flat template of shoe-lasts

The basic pattern of flat-knitted shaped uppers is drawn and revised on the basis of flattened shoe last pattern in the ratio method and made for knitting on flat knitting machine, based on the characteristics of human's feet and shoe last structures and the flat knitting principle. It can be seen in Table 1 for the common design sizes of jogging shoes.

Table 1. The key value range (foot length 255)

\begin{tabular}{|c|c|c|c|c|}
\hline Style & $\begin{array}{c}\text { Heel } \\
\text { height }\end{array}$ & $\begin{array}{l}\text { Ankle } \\
\text { height }\end{array}$ & $\begin{array}{c}\text { Foot } \\
\text { mountain } \\
\text { height }\end{array}$ & $\begin{array}{l}\text { Opening } \\
\text { width }\end{array}$ \\
\hline $\begin{array}{l}\text { Low-top } \\
\text { shoes }\end{array}$ & $75-86$ & $58-70$ & $90-100$ & \multirow{3}{*}{$12-18$} \\
\hline $\begin{array}{l}\text { Medium-top } \\
\text { shoes }\end{array}$ & $88-95$ & \multirow{2}{*}{$\begin{array}{c}\text { Over the } \\
\text { ankle } \\
\text { bone }\end{array}$} & $115-125$ & \\
\hline $\begin{array}{l}\text { High-top } \\
\text { shoes }\end{array}$ & 120 & & $\geq 120$ & \\
\hline
\end{tabular}

Table 1 shows the reference value range of the corresponding shoe parts, and a set of specific data is selected in the practical application.

Drawing a contour line along the shoe's last pattern, get front point $\mathrm{J}$, then finding the midpoint of heel position, get rear point A1, segment JA1 as the whole length of shoe last preface. Determine the entrance point $E$ at $31 \%$ of the length of JA1, the upper height position point $d$ at $63 \%$ of the length of JA1, remaining $37 \%$ JA1 for upper posterior segment length. Taking into account that shoes on the feet should have a certain cohesion power but cannot prevent ankle activity during the process of running, the width of the opening EF is designed to $15 \mathrm{~mm}$, counter height $A A^{\prime} 80 \mathrm{~mm}$, the height of ankle BB' is designed to $65 \mathrm{~mm}$, mid-height DD' $100 \mathrm{~mm}$.

During the pattern of magnifying process, feet length increase of 1 degree increase heel height $1 \mathrm{~mm}$, ankle height $0.5 \mathrm{~mm}$, foot mountain $2 \mathrm{~mm}$. Design templates should be made taking into account the sole technique, take lasting technology as 


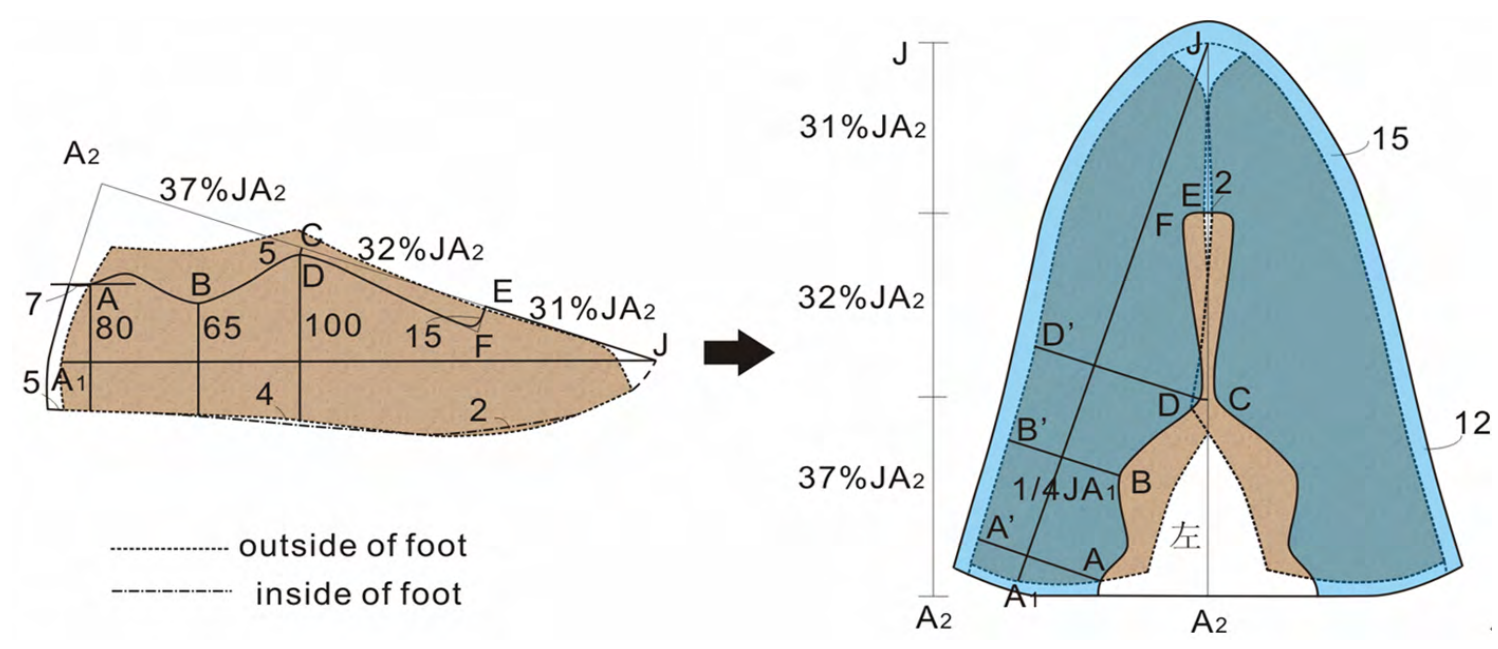

Figure 3. Making basic pattern of flat knitting shaping shoe-upper by ratio method

an example. From A1 and A reserved 5 and $7 \mathrm{~mm}$ as a foam thickness in the heel, top half outlines increase $15 \mathrm{~mm}$, lower half outline increase $12 \mathrm{~mm}$, smooth the outline then can be obtained the shoe last pattern. The basic pattern of flat knitted shaped upper can be obtained after the adoption of rotation and marked dimensions.

\subsection{Implementation of flat-knitted half-shaped uppers}

The flat-knitted half-shaped uppers were made after the achievement of outer contour changes with flat knitting technology and cutting processing of contours in the top-lines, with the pattern of uppers as prototypes. As shown in Figure 6 , with the pattern of upper as the prototype, the outer contours of half-shaped uppers can be obtained by means of narrowing and local knitting, mark the contours of cutting area by means of transfer, the jacquard technology can be used to mark the label in the cutting area, and finally shape the uppers by tailoring the shaded area. The advantages of the technology lie in simple shaping methods with only the shaping design of outer contour and efficient production from common computerised flat knitting machines. With the technology, diverse structures and patterns will be achieved, as well as breathable function for uppers and efficient production of uppers products through the matching design of different structures. Meanwhile, the diversification of colours meets the requirements of people on decorative garment accessories.

\subsection{Implementation of flat-knitted fully shaped uppers}

The flat-knitted fully shaped uppers are those that can be knitted in the flat knitting machines without cutting, finally obtained after shear deformation of basic uppers pattern. The design for basic pattern of flat knitted uppers provides a direct basis for the design and production of shaped uppers. Since the flat knitted fabrics are highly elastic, the selection of raw materials and equipment are restricted to a certain degree, so that the flat-knitted shaped uppers can meet the requirements on uppers materials.

Take the left half of the upper pattern as an example. First, mark the front opening end (Point a), the highest point of topline (Point $c$ ) and the heel end (Point $\mathrm{f}$ ), make the Straight Lines ("ab" and "cd") via Point a and Point c, perpendicular to the topline and crossing the outer contour line of topline pattern at Point $b$ and Point $d$, and make the tangent line of shoe neckline

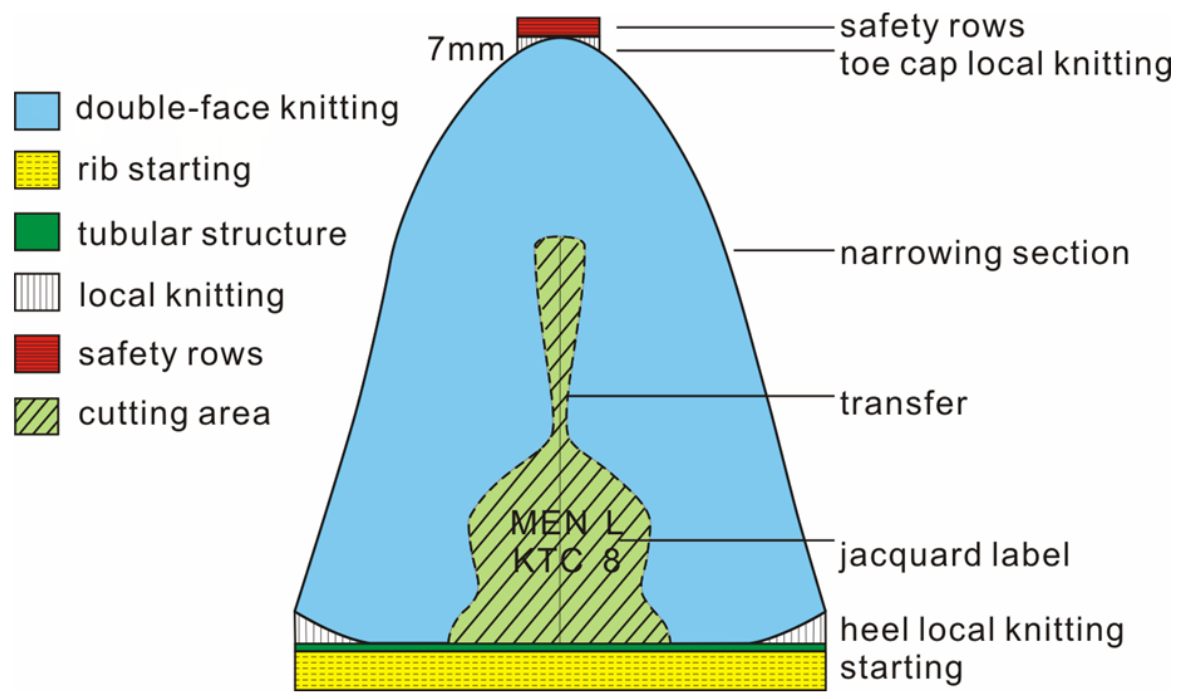

Figure 4. Knitting technology of flat-knitted half-shaped upper 
at Point $f$ which crosses at Point e; cut off "ab", "cd" and "ef" along the vertical Straight Line $L$ via Point a, rotate "ab" around the fixed Point b, and get "a1b" perpendicular to L; rotate "cd" around Point $\mathrm{d}$ so that Point $\mathrm{c}$ can fall in the midpoint extension line of "c1f1" formed by Point $\mathrm{c} 1$ and Point $\mathrm{f} 1$ (the intersection point of vertical Line $L$ and horizontal Line ff1). Then rotate "ef" around Point $f$ so that the e1f1 formed by Point e1 (the intersection point made by the extension line of Point $e$ and the vertical Line $L$ ) and Point f1 can have the ratio of 3:2 with the e1g1 formed by Point e1 and Point g1 (the intersection point made by the extension line of Point $g$ and the vertical Line $L$ ), and adjust the uppers pattern (the right and left pattern are adjusted in the same way); finally, the adjusted pattern can be used as the knitting pattern of fully-shaped uppers, as shown in Figure 5.

As shown in Figure 6, the requirements on girth surrounding human heel are met by means of widening, and the difference between inner and outer lower openings of uppers is achieved by means of open left-right asymmetry narrowing, which meets the difference requirements of longitudinal arch sizes and bending radians on both inner and outer sides of human's feet. In the knitting process, the part below the entrance opening is removed, based on the number of needles throughout the width of entrance opening, the separate knitting of front opening sneaker uppers is achieved. Meanwhile, the blank part is filled by means of local knitting; to achieve the girth surrounding the front tarsus of human's foot and the curved shape from entrance opening to instep. Large area of the rear uppers is knitted to achieve the curve radian at the heel, but due to excessively large local edge range in the area, uneven stretch, broken line and other problems occur in the knitting process. So two rows or four rows are added in the middle of the local edge in the area for transition, in order to facilitate better production.

The starting opening of adjusted pattern is maintained horizontal, so the beginning part of fabrics and the shoe neckline rib part can be knitted normally, not only achieving the one-time shaping of knitting, but also forming certain thickness of rib part by plating and other knitting ways, meeting the stiffness requirements from the sneaker uppers to necklines, playing a good support and protection role for the ankles, saving the raw materials, simplifying the processing steps of shoe materials, reducing labour costs, and reducing the environmental pollution to a certain degree. However, the knitting process in the shaping way is complicated and takes a long time, and has higher technical requirements on rolling

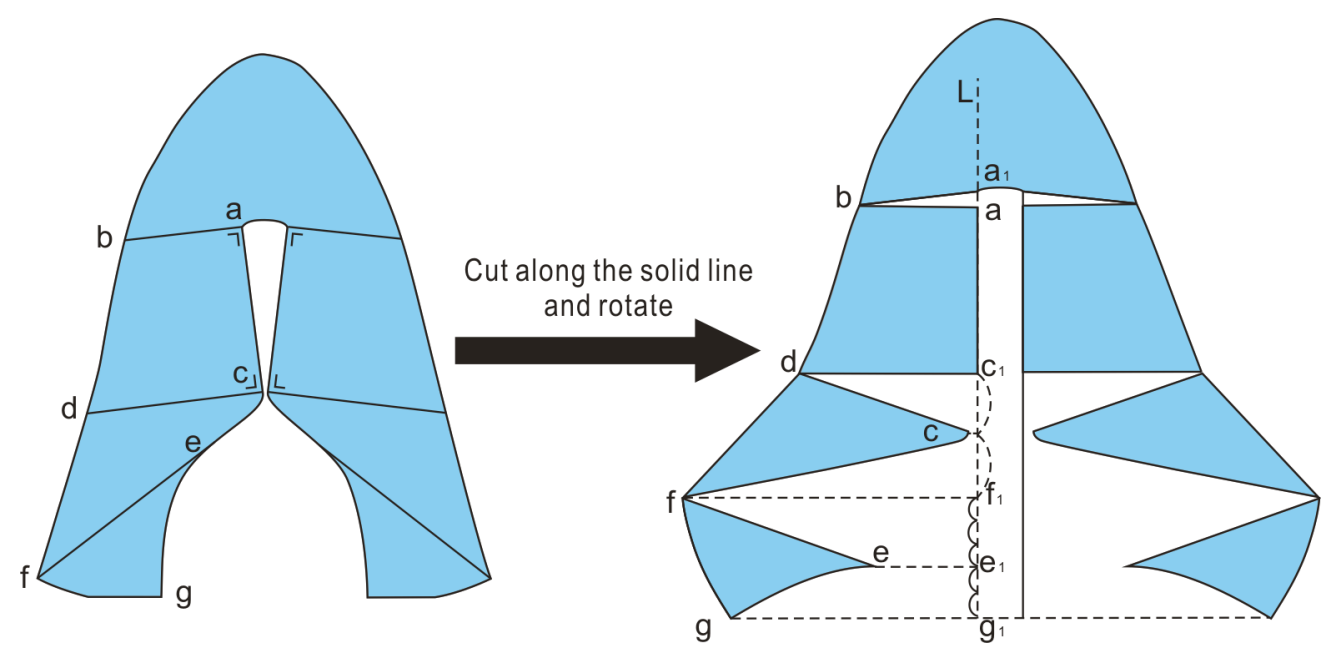

Figure 5. Pattern's deformation of fully-shaped upper

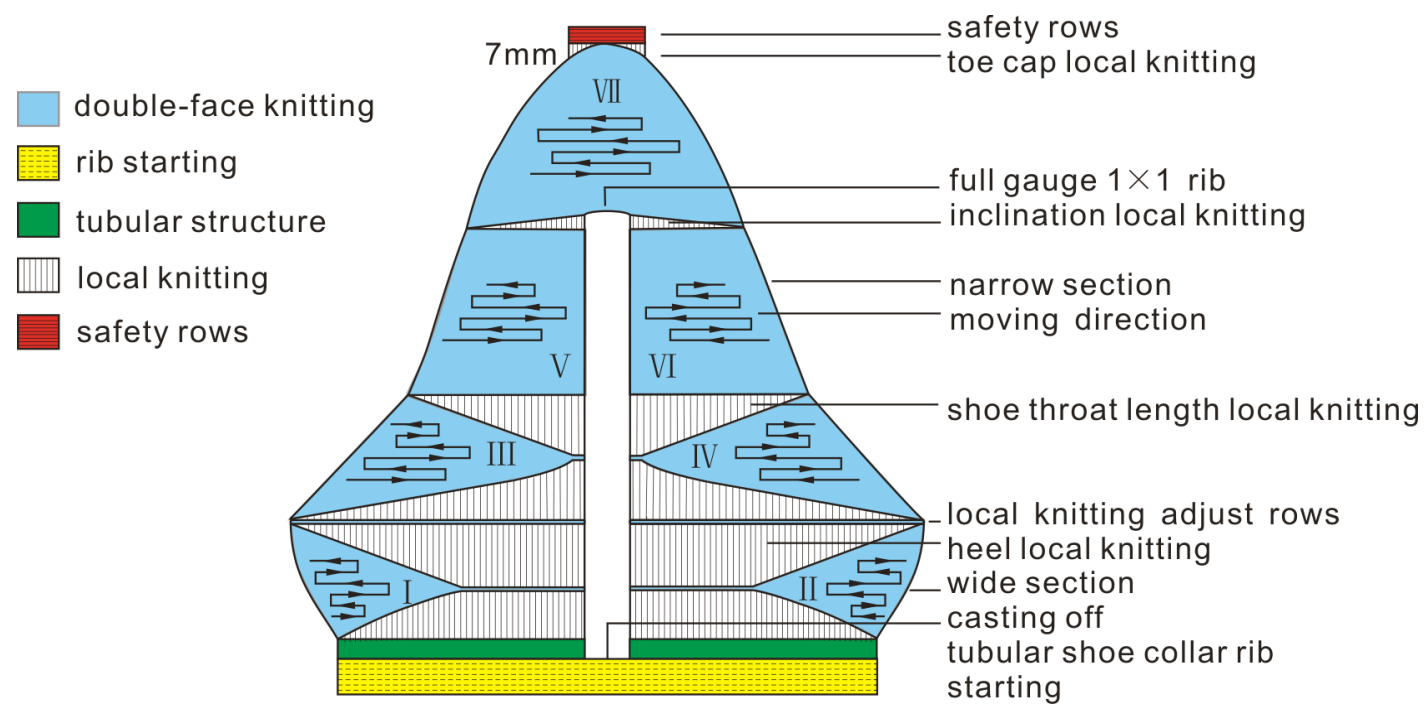

Figure 6. Knitting technology of flat knitted fully-shaped upper 
performance of machine and lower production efficiency, so it still needs further study on how to improve the production efficiency of fully-shaped uppers.

\section{The structure and technology of flat-knitted shaped uppers}

The wearability is closely interrelated to the stitch of flat knitting uppers, in order to meet people's requirements for trainers of functional and decorative, it is very important to research on the stitch. The flat-knitted shaped uppers are obtained by the reorganization of looping, tucking, floating, transferring, racking and other process modules according to the knitting requirements, to form the fabric structure with certain arrangement rules and the wearability of upper materials, achieving the complete shaping of uppers materials. Two designs (namely, functional design and decorative design) are proposed to for the organizational structures of shaped uppers, to better exert their shaping advantages, meet the wearability requirements of upper materials, and achieve good decorative effect.

\subsection{Functional structure}

The functional design of flat knitted uppers mainly covers the enhancement design and improvement design of strength, thickness and stiff and smooth performance of uppers, by means of changes on organizational structure.
The enhanced structure mainly covers the looping structure of tubular stitch and reinforcing rib structure of vertical side, as shown in Figure 7 and Figure 8 . Figure 7 is the tubular stitch combined with tucked loops which are knitted by other yarns in the tubular stitch, which not only increased the thickness of fabrics, but also achieved stiff effect of uppers. It is designed mainly to meet the thickness requirements on flat knitted uppers which are taken as the upper materials, enhance the thermal effect, provide more comfortable wearing environment, and play a cushioning role during walk or exercise. The reinforced rib structure, with an interlock stitch as the basic structure, the tubular stitch with only front loop is added which is formed by local knitting, thus, the hollow structure can be obtained with a certain gap through which a supporting strip can cross, the ergonomics requirements can be met, and the supporting effect from uppers to feet will be achieved.

The functional improvement design of flat knitted uppers can be achieved through roughness breathing holes structure and top-line rib structure. As shown in Figure 9, it is roughness breathing holes structure knitted directly by computerized flat knitting machine with the Milano rib as the basic structure. The breathing holes structure similar to a square structure can be formed through flat needle bed transfer and looping by organizing and knitting it with the configuration of three lines of tubular stitch and a line of interlock stitch, and moving two adjacent loops of front needle bed on the tubular stitch to the rear needle bed, respectively, and make correlation again through interlock rib to avoid the loop laddering in the horizontal line of tubular stitch, which not only reduces material wastes


Figure 7. Tubular stitch with tucked loop
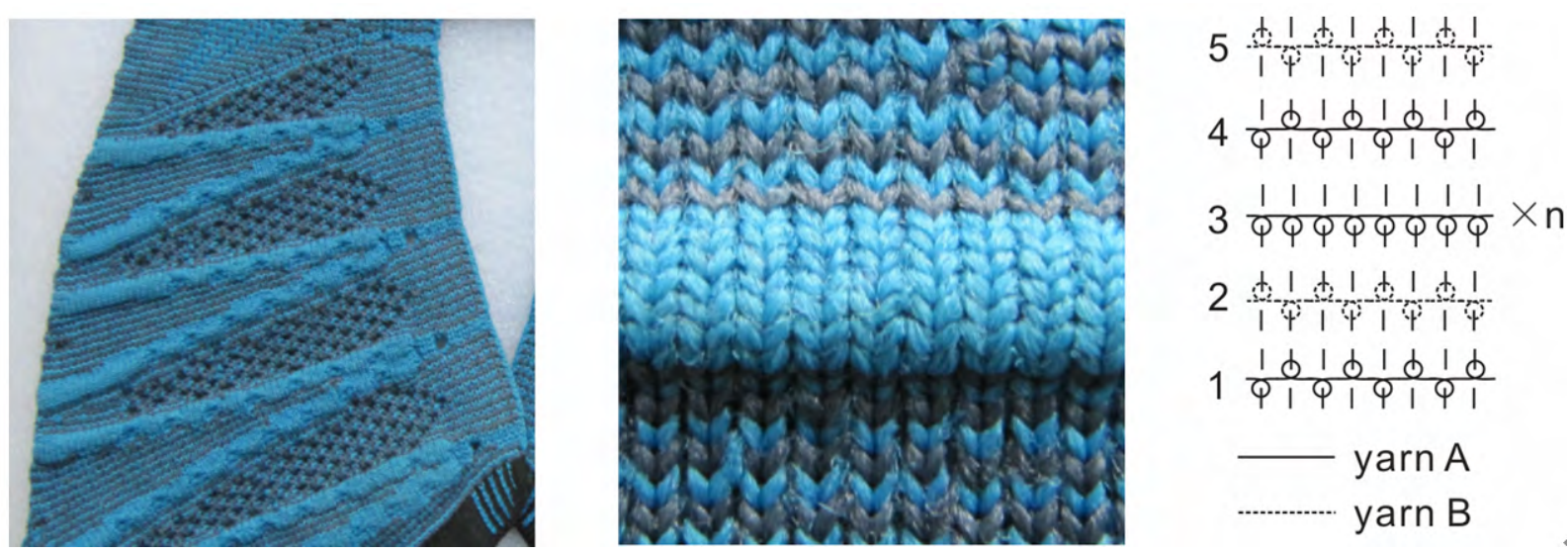

Figure 8. Reinforced rib structure 
but also achieves good moisture absorption and permeability performances, compared with the breathing holes of uppers formed in the normal punching way. Through the selection of yarn and the knitting technology, the flat knitting uppers could satisfy the requirement of function of sports shoes, at the same time, enhanced with the comfort level of foot skin tactility [14].

The top-line rib structure is a rib structure with the tightened function and used at the shoe neckline. As shown in Figure 10, a tricolour float jacquard tubular stitch is formed with the tubular stitch as the basic structure and combining the float jacquard technology. An elastomeric yarn should be added for joint knitting process, to achieve not only good tensile property and rebound effect but also a curve in the natural state. Meanwhile, it achieves tricolour interval effect on the fabric surface, increases the thickness of fabric and has certain decorative effect at the same time of meeting the functional requirements on shoe necklines, playing a good tighten role for feet, and ensuring safety in sports.

\subsection{Decorative structure}

As for the flat-knitted shaped uppers with both functional and decorative characteristics, as shown in Figure 11, the coloured silk knitting and changed structure are taken for the decorative design, so that the flat knitted uppers can be characterized by rich colours and diverse structure changes. The effect with dots or strips on the fabric surface by means of yarn knitting of different colours, not only meet the decorative requirements on shoe materials, but also reduce the follow-up dyeing process and achieve energy conservation and environmental protection. Meanwhile, in the knitting process, the knitting of


Figure 9. Breathing holes structure
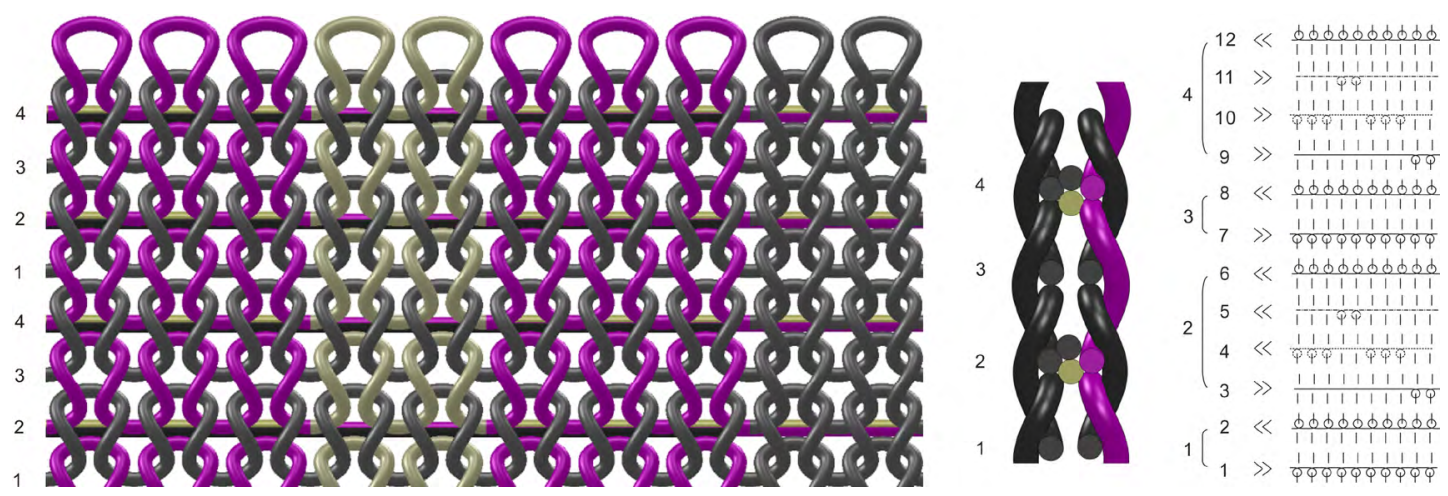

Figure 10. Topline rib
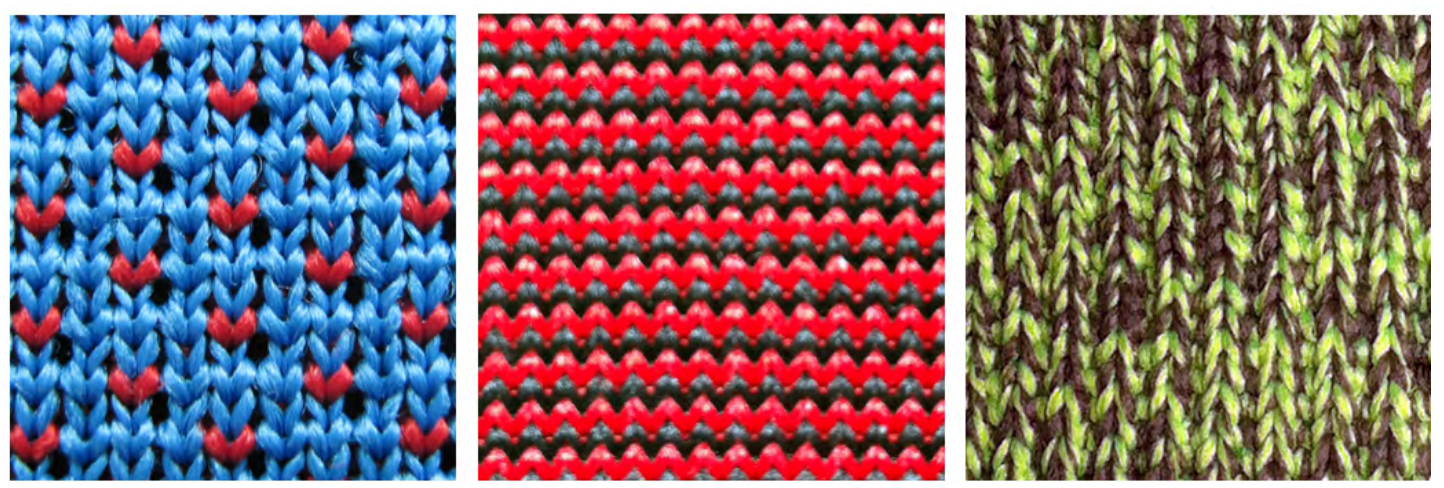

Figure 11. Decorative flat-knitted structure. 
flat-knitted shaped uppers with the section-dyed yarn and other fancy yarns as raw materials can better improve the fancy effect of products and increasing the artistic beauty of products.

\section{Design example}

The STOLL CMS530 E7.2 computerized flat knitting machine was selected to produce the flat-knitted shaped uppers. The actual knitting speed in the experiment was $0.7 \mathrm{~m} / \mathrm{s}$. $700 \mathrm{D}$ light-green and 700D dark-green spun polyester yarns were employed in this application example.

The corresponding relationship between the size and process model for the basic pattern of flat-knitted shaped uppers was as follows:

The number of started needles $=\mathrm{P}_{\mathrm{A}}($ Wales per centimetre $) \times$ shoe top-line size

The number of horizontal lines of uppers $=P_{B}$ (Courses per centimetre) $\times$ shoe length

In combination with the PA and PB, the flat-knitted shaped upper pattern was obtained through correction. In the process design, the flat-knitted shaped uppers were achieved by half-shaped and fully-shaped ways, respectively. In the M1 pattern preparation system, the basic pattern was inputted with the number of stitches and rotations converted into the shape editor, conducting the process design and parameter modification based on the styles, organizational structure and pattern effects to meet the requirements of shaping uppers, and finally export it from the software and convert it into the documents of ".sin", ".set" and ".jac" formats for knitting on the machine. The flat-knitted shaped shoe products obtained finally as shown in the figure 12 .
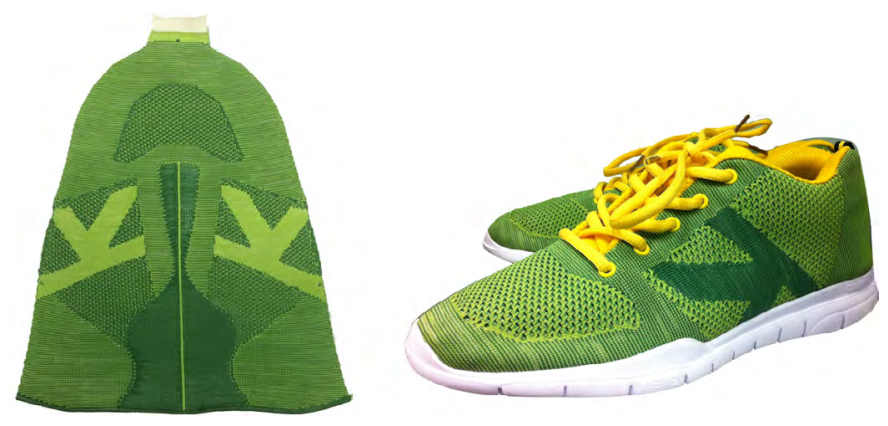

Figure 12. Flat-knitted half-shaped uppers and shoe

\section{Conclusions}

In this paper, revised on the basis of flattened shoe last in the ratio method, the basic pattern of flat-knitted shaped uppers is drawn. In the application process of the basic pattern, flat knitting and shaping principle were used ,the knitting method of half-shaped and fully-shaped uppers were obtained, the flat knitted uppers were designed and produced, achieving the connection of process and design.
Based on the wearability of the upper materials, two designs (namely, functional design and decorative design) are proposed for the structures of shaped uppers. At the same time of exerting its advantages in shaping process, the functional, decorative and stable and efficient knitting production are achieved on the premise of ensuring process feasibility by practicing the typical organizational structure for shaped uppers on the computerized flat knitting machine.

Through yarn selection, model design and knitting structure design, the flat knitted uppers satisfy the human foot structures and physiological functions, including regulating temperature, secreting sweat and evaporation, and are also flexible, provide permeability, comfort and warmth retention property requirements. This paper puts forward the new design idea for sports shoes and provides a new approach for the production.

\section{Acknowledge}

The authors acknowledge the financial support from the National Science Foundation of China (No.11302085 and 51403080), the Fundamental Research Funds for the Central Universities (No. JUSRP1043 and JUSRP51404A) and the Innovation fund project of Cooperation among Industries, Universities \& Research Institutes of Jiangsu Province (No. BY2014023-34 and BY2014023-20).

\section{References}

[1] ÖZDIL N, ANAND S. Recent Developments in Textile Materials and Products Used for Activewear and Sportswear[J]. Tekstil Teknolojileri Elektronik Dergisi, 2014, 8(3): 68-83.

[2] CHOI, W. and N. B. POWELL. Three dimensional seamless garment knitting on $\mathrm{V}$-bed flat knitting machines[J]. Journal of Textile and Apparel, Technology and Management, 2005,4(3): 1-33.

[3] GOKARNESHAN, N., B. VARADARAJAN, et al. Engineering knits for versatile technical applications: Some insights on recent researches[J]. Journal of Industrial Textiles, 2012,42(1): 52-75.

[4] HONG, H., A. FILHO, et al. The development of $3 D$ shaped knitted fabrics for technical purposes on a flat knitting machine[J]. India Journal of Fibre and Textile Research, 1994, 19: 189-189.

[5] ABOUNAIM, M. D. and C. CHERIF. Flat-knitted innovative three-dimensional spacer fabrics: a competitive solution for lightweight composite applications[J]. Textile Research Journal, 2012, 82(3): 288-298.

[6] SONG, G., J. WU, et al. Computer-aided design of threedimensional knitwear[J]. Journal of the Textile Institute, 2006, 97(6): 549-552.

[7] BARTON, C. J., D. BONANNO, et al. Development and evaluation of a tool for the assessment of footwear characteristics[J]. Journal of Foot and Ankle Research, 2009, 2.

[8] Ciesielska-Wrobel I L, Van Langenhove L, Grabowska K. Fingertip skin models for analysis of the haptic perception of textiles[J]. Journal of Biomedical Science and Engineering, 2014, 7:1-6. 
[9] SCOTT, S. H. and D. A. WINTER. Biomechanical model of the human foot: kinematics and kinetics during the stance phase of walking[J]. Journal of biomechanics, 1993, 26(9): 1091-1104.

[10] MUNDERMANN, A, B. M. NIGG, et al.. Development of a reliable method to assess footwear comfort during running[J]. Gait \& posture, 2002, 16(1): 38-45.

[11] JONES, B. H., M. M. TONER, et al. The energy cost and heart-rate response of trained and untrained subjects walking and running in shoes and boots[J]. Ergonomics, 1984, 27(8): 895-902.
[12] NISHIWAKI, T. Requirement Properties and Materials Used in Sport Shoes[J]. Sen-I Gakkaishi, 2009, 65(5): P150-P153.

[13]XIONG, S., R. S. GOONETILLEKE, et al. Modelling foot height and foot shape-related dimensions[J]. Ergonomics, 2008, 51(8): 1272-1289.

[14] Ciesielska-Wrobel I L, Van Langenhove L. The hand of textiles- definitions, achievements, perspectives- a review[J]. Textile Research Journal, 2012, 82(14): 14571468. 